University of Wollongong

Research Online

Faculty of Engineering and Information

Faculty of Engineering and Information

Sciences - Papers: Part A

Sciences

$1-1-2016$

Online coordinated voltage control in distribution systems subjected to structural changes and DG availability

Dothinka Ranamuka Rallage

University of Wollongong, dssrr987@uowmail.edu.au

Ashish P. Agalgaonkar

University of Wollongong, ashish@uow.edu.au

Kashem M. Muttaqi

University of Wollongong, kashem@uow.edu.au

Follow this and additional works at: https://ro.uow.edu.au/eispapers

Part of the Engineering Commons, and the Science and Technology Studies Commons

Research Online is the open access institutional repository for the University of Wollongong. For further information contact the UOW Library: research-pubs@uow.edu.au 


\title{
Online coordinated voltage control in distribution systems subjected to structural changes and DG availability
}

\begin{abstract}
The responses of multiple DG units and voltage regulating devices such as tap changers and capacitor banks for correcting the voltage may lead to operational conflicts and oscillatory transients, where distribution systems are subjected to network reconfiguration and changes in availability of the DG units. Therefore, coordinated voltage control is required to minimize control interactions while accounting for the impact of structural changes associated with the network. This paper proposes a strategy for coordinating the operation of multiple voltage regulating devices and DG units in medium voltage (MV) distribution systems, under structural changes and DG availability, for effective voltage control. The proposed strategy aids to minimize the operational conflicts by allowing the farthest voltage regulating device to operate first on a priority scheme designed based on the electrical-distance between voltage regulating devices and DG units, while maximizing the voltage support by the DG units. The proposed coordination scheme is designed to enact with an aid of a substation centered distribution management system (DMS) for online voltage control. The control actions of proposed coordination strategy are tested on a MV distribution system, derived from the state of New South Wales, Australia, through simulations, and results are reported.
\end{abstract}

\section{Keywords}

voltage, control, availability, distribution, systems, subjected, structural, changes, online, dg, coordinated

\section{Disciplines}

Engineering | Science and Technology Studies

\section{Publication Details}

D. Ranamuka Rallage, A. P. Agalgaonkar \& K. M. Muttaqi, "Online coordinated voltage control in distribution systems subjected to structural changes and DG availability," IEEE Transactions on Smart Grid, vol. 7, (2) pp. 580-591, 2016. 


\title{
Online Coordinated Voltage Control in Distribution Systems Subjected to Structural Changes and DG Availability
}

\author{
D. Ranamuka, Student Member, IEEE, A. P. Agalgaonkar, Senior Member, IEEE, and K. M. Muttaqi, \\ Senior Member, IEEE
}

\begin{abstract}
The responses of multiple DG units and voltage regulating devices such as tap changers and capacitor banks for correcting the voltage may lead to operational conflicts and oscillatory transients, where distribution systems are subjected to network reconfiguration and changes in availability of the DG units. Therefore, coordinated voltage control is required to minimize control interactions while accounting for the impact of structural changes associated with the network. This paper proposes a strategy for coordinating the operation of multiple voltage regulating devices and DG units in medium voltage (MV) distribution systems, under structural changes and DG availability, for effective voltage control. The proposed strategy aids to minimize the operational conflicts by allowing the farthest voltage regulating device to operate first on a priority scheme designed based on the electrical-distance between voltage regulating devices and DG units, while maximizing the voltage support by the DG units. The proposed coordination scheme is designed to enact with an aid of a substation centered distribution management system (DMS) for online voltage control. The control actions of proposed coordination strategy are tested on a MV distribution system, derived from the state of New South Wales, Australia, through simulations, and results are reported.
\end{abstract}

Index Terms--control coordination; distributed generation; distribution system; network reconfiguration; on-load tap changer; step voltage regulator; voltage control.

\section{NOMENCLATURE}

$\begin{array}{ll}\text { OLTC } & \text { On-load tap changer } \\ \text { SVR } & \text { Step voltage regulator } \\ \text { CB } & \text { Capacitor bank } \\ \text { DG } & \text { Distributed generation } \\ \text { MV } & \text { Medium voltage } \\ \text { PV } & \text { Photovoltaic } \\ \text { DMS } & \text { Distribution management system } \\ \text { ABS } & \text { Air break switch } \\ s & \text { State of any system configuration } \\ t & \text { Time } \\ T a p_{S t} & \text { SVR tap position } \\ V_{S L C} & \text { SVR target point voltage } \\ V_{L B} & \text { SVR lower boundary voltage } \\ V_{U B} & \text { SVR upper boundary voltage }\end{array}$

The authors are with the Australian Power Quality and Reliability Centre, School of Electrical, Computer and Telecommunications Engineering, University of Wollongong, New South Wales 2522 Australia. (e-mail: dssrr987@uowmail.edu.au; ashish@uow.edu.au; kashem@uow.edu.au)

\begin{tabular}{|c|c|}
\hline$V_{S E T / V R}$ & SVR voltage set value \\
\hline$D B$ & SVR dead-band limit \\
\hline$S F C_{s t}$ & CB switching position \\
\hline$V_{S F C}$ & CB target point voltage \\
\hline$V_{O N}$ & CB switching $O N$ voltage \\
\hline$V_{O F F}$ & CB switching $O F F$ voltage \\
\hline$M_{\mathrm{SVP}}$ & $\begin{array}{l}\text { System matrix of partial derivatives, } \\
\delta V / \delta P \text { where } V \text { is voltage and } P \text { is active } \\
\text { power }\end{array}$ \\
\hline$M_{s V Q}$ & $\begin{array}{l}\text { System matrix of partial derivatives, } \\
\delta V / \delta Q \text { where } Q \text { is reactive power }\end{array}$ \\
\hline$J$ & System Jacobian matrix \\
\hline$V_{\text {si/by } D G}$ & Voltage change at $i^{\text {th }}$ node by DG output \\
\hline$P_{S D G}$ & DG active power \\
\hline$Q_{s D G}$ & DG reactive power \\
\hline$\alpha_{s}$ & Phasor angles, $\alpha$ of nodal voltages \\
\hline$M_{s \alpha P}$ & $\begin{array}{l}\text { System matrix of partial derivatives, } \\
\delta \alpha / \delta P\end{array}$ \\
\hline$M_{s \alpha Q}$ & $\begin{array}{l}\text { System matrix of partial derivatives, } \\
\delta \alpha / \delta Q\end{array}$ \\
\hline$\mu_{s i j}$ & $\begin{array}{l}\text { Attenuation of voltage variation between } \\
\text { node } i \text { and } j\end{array}$ \\
\hline$D_{s i j}$ & Electrical distance between nodes $i$ and $j$ \\
\hline$a_{i j}$ & Elements of $M_{S V Q}$ matrix \\
\hline $\mathrm{N}_{\mathrm{dg} 1}$ & DG1 nodal bus \\
\hline $\mathrm{N}_{\mathrm{dg} 2}$ & DG2 nodal bus \\
\hline $\mathrm{N}_{\mathrm{a}}, \mathrm{N}_{\mathrm{b}}$ & End nodes of DG1 voltage control zone \\
\hline $\mathrm{N}_{\mathrm{c}}, \mathrm{N}_{\mathrm{d}}$ & End nodes of DG2 voltage control zone \\
\hline$V_{s L C(m)}$ & Target point voltage of any $m^{\text {th }}$ SVR \\
\hline$V_{s L C(m) / b y} D G$ & $\begin{array}{l}\text { Voltage change at target point of any } m^{\text {th }} \\
\text { SVR by DG reactive power output }\end{array}$ \\
\hline$V_{S F C(g)}$ & Target point voltage of any $g^{\text {th }} \mathrm{CB}$ \\
\hline$V_{s C B(g)}$ & CB connection nodal voltage \\
\hline$V_{S F C(g) / b y ~ D G}$ & $\begin{array}{l}\text { Voltage change at target point of any } g^{\text {th }} \\
\text { CB by DG reactive power output }\end{array}$ \\
\hline
\end{tabular}

\section{INTRODUCTION}

$\mathrm{T}$ HE conventional voltage regulation by means of multiple voltage regulating devices such as OLTCs, SVRs and CBs may need to be operationally updated in the presence of DG units. Both, simultaneous and non-simultaneous responses of DG units and multiple voltage regulating devices may lead to conflicting operations, resultant voltage variations and voltage rise in the system, especially in case if DG units 
operate in voltage control mode. Based on the inherent operational characteristics of DG units and voltage regulating devices for correcting the target bus voltage, both simultaneous and non-simultaneous responses of voltage regulating devices and DG units are existed. If there are tap operations for OLTC and SVRs, switching operations for CBs and DG responses at the same time; then their cumulative effect can exceed the nodal voltage beyond maximum allowable voltage limit for the distribution system or vice versa, thereby violating the dead-band values of voltage regulating devices in opposite direction. Hence, the simultaneous responses of voltage regulating devices and DG units can lead to conflicting operations under certain system operating conditions and may exhaust total number of tap operations resulting into voltage variations. Similarly, the nonsimultaneous responses of voltage regulating devices and DG units for correcting the target bus voltage can also lead to the conflicting operations based on the impedance between voltage regulating devices and DG units, DG penetration level and their control parameters such as voltage set values, time delays and dead-band limits. This can be more complex phenomena, where the distribution systems are subjected to structural changes and the availability of DG units. The proposed method in this paper deals with the distribution system operation. Therefore, the system changes are the structural changes resulted by seasonal and outage caused reconfigurations. Also, availability of DG units depends on the active and reactive power dispatch requirements of the network operator, adequacy of prime resources, and preventive and breakdown maintenance of DG units, which also may lead to topological changes in the overall system. For example, significant topological changes may be occurred due to the shutdown and/or energizing DG units in multiple locations which are connected to the medium voltage distribution system using considerably long feeders emerging out of substations.

One of the feasible ways of minimizing the adverse effects of interactions among DG units and voltage regulating devices is control coordination. In [1], an online voltage control strategy is proposed in order to operationally avoid the impact of simultaneous responses of multiple voltage regulating devices and voltage support DG units in real time using a DMS based signal processing scheme. However, it does not address how the impacts of non-simultaneous operations of DG units and voltage regulating devices are minimized. Also, the rule and optimization based strategies proposed in [2], [3], [4], [5], [6] for maximizing DG voltage support and coordinating the voltage regulating devices and DG units do not clearly address how the proposed controls operationally perform for minimizing the impact of DG and voltage regulating device interactions when the distribution systems are subjected to structural changes and DG availability. There may be topology based limitations, which are clearly discussed in [2]. In [7], the $\varepsilon$-decomposition and deep first search methods are used to find out the network bus voltages which are significantly influenced by DG units in order to utilize multiple DG units for coordinated voltage control. Similarly, power flow sensitivity factor based strategies for coordinated output control of multiple DG units in [8]. In [9], [10], on-line coordinated voltage control strategies are proposed for effective voltage control in distribution systems under DG rich environment. However, these methods do not address a way for coordinating the DG units with other voltage regulating devices in case of distribution systems with multiple voltage regulating devices and multiple DG units. In [11], a strategy is proposed for optimal voltage control and coordination with distributed generation in distribution systems with multiple voltage regulating devices and DG units. It is formulated as a large-scale optimization problem, where the objective function is set up so that a voltage margin can be secured to a steep voltage variation while minimizing power losses. The amount of operation by each voltage control device is determined using a genetic algorithm based solver. Similarly, a strategy is proposed for coordinated control of DG units and voltage regulating devices using a dynamic programming algorithm in [12]. However, there is no guarantee or mechanism to minimize the DG unit and voltage regulating device interactions after enacting the dispatched local control actions of voltage regulating devices and DG units at $t=t$ until the next dispatch decision is enacted at $t=t+1$.

The proposed coordination strategy in this paper is designed to alleviate above technical issues for effective voltage control in MV distribution systems with multiple tap changers, capacitor banks and DG units which are operated for maximizing the voltage support. The proposed strategy aids to operate voltage regulating devices and DG units on priority basis and thereby minimizing the possible interactions and the resultant adverse effects. Since, the control decisions are based on the electric distance among the DG units and the voltage regulating devices, the proposed strategy is able to effectively account for the impact of structural changes associated with the network. Also, it exhibits the feature to use a substation centered DMS for online voltage control.

This paper is organized as below. Section II outlines the theoretical background of the proposed methodology and Section III contains the algorithm of the proposed coordination strategy. Section IV elaborates realistic case study while Section $\mathrm{V}$ includes the concluding remarks.

\section{Deriving Voltage Control Zones of DG IN DISTRIBUTION SYSTEMS}

Modern Distribution systems may contain multiple voltage regulating devices for voltage control and can be subjected to structural changes due to network reconfiguration and availability of DG units embedded in the network. A typical distribution system is shown in Fig. 1. In accordance with the operation of tie-lines, with the aid of related ABSs, and network reconfiguration, there could be multiple feeder systems, which may be subjected to structural changes. Moreover, if such a feeder system contains multiple DG units, further topological changes are possible depending on the availability of the DG units as discussed in Section I. 


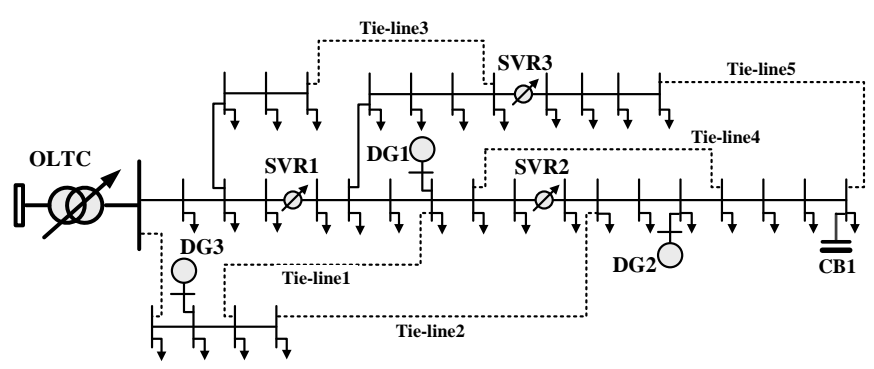

Fig. 1. Topology of a distribution system capable of operating under structural changes with multiple voltage regulating devices.

In each system configuration, the voltage magnitude at $i^{\text {th }}$ node of the distribution system for small changes in active and reactive power injections by a DG unit can be obtained using $M_{S V P}$ and $M_{S V Q}$ matrices which are derived from the system Jacobian matrix as given by (01). It is assumed that the DG unit is connected at node- $k$ for the MV distribution system with $n$ number of nodes, which are numbered chronologically from the distribution substation-end.

$$
\begin{gathered}
\Delta V_{s i / b y D G}=\left(M_{s V P}\right)_{s i, k} \times \Delta P_{s D G}+\left(M_{s V Q}\right)_{s i, k} \times \Delta Q_{s D G} \\
{\left[\begin{array}{l}
\Delta \alpha_{s} \\
\Delta V_{s}
\end{array}\right]=\left[J^{-1}\right] \times\left[\begin{array}{c}
\Delta P_{s} \\
\Delta Q_{s}
\end{array}\right] \quad \text { where } \quad J^{-1}=\left[\begin{array}{l|l}
M_{s V P} & M_{s V Q} \\
\hline M_{s V P} & M_{s V Q}
\end{array}\right]}
\end{gathered}
$$

The term associated with active power injection by a DG unit, $\left(M_{s V P}\right)_{s i, k} \mathrm{x} \triangle P_{s D G}$ depends on the type of DG technology, primary energy source, the system topology and its operation. However, the term associated with reactive power injection, $\left(M_{s V Q}\right)_{s i, k} \mathrm{x} \triangle Q_{s D G}$ can be controlled for the overall system voltage support. Also, voltage control zone for such a DG unit can be derived as follows.

The magnitude of voltage coupling between any two busses of a power system can be quantified by the maximum attenuation of voltage variation between the two busses. The respective attenuations can be derived from $\left[M_{s V Q}\right]$ matrix by dividing the elements of each column by the diagonal term [13], [14]. Therefore, the matrix of attenuations, $\left[\mu_{s i j}\right]$ among all the busses of the power system can be derived as shown in (02). The electrical distance between nodes $i$ and $j$ is given by (03). The normalized electrical distance is given by (04).

The step by step methodology for deriving the voltage control zones for multiple DG units is given below.

$$
\begin{array}{r}
{\left[\Delta V_{s}\right]=\left\lfloor M_{s V Q}\right\rfloor_{n \times n} \times\left[\Delta Q_{s}\right] \quad \text { and } \Delta V_{s i}=\mu_{s i j} \times \Delta V_{s j}} \\
\mu_{s i j}=\frac{\partial V_{s i}}{\partial Q_{s j}} / \frac{\partial V_{s j}}{\partial Q_{s j}} \text { and } \mu_{s i j} \neq \mu_{s j i}
\end{array}
$$

Step-I: Calculate the system Jacobian matrix, $J$ and derive its inverse matrix, $J^{-1}$.

Step-II: Obtain $\left[M_{s V Q}\right]$ matrix, and the elements of this matrix are written as $a_{i j}=\left(\partial V_{s i} / \partial Q_{s j}\right)$.

Step-III: Obtain the attenuation matrix $\left[\mu_{s i j}\right]$, and the elements of this matrix are written as $\mu_{s i j}=\left(a_{s i j} / a_{s j j}\right)$. (5).

Step-IV: Calculate the electrical distance, $D_{s i j}$ array using

Step-V: Normalize the electrical distance values using (06).

Step-VI: For each DG unit bus, its normalized electrical distances to all other buses $(j, j \neq i)$ are classified into different pre-specified ranges such as Range1, Range2, Range3, ..., which are ranked in ascending order using $R 1, R 2$ and $R 3$ parameters as given below. This is done for the sake of convenience in traversal and construction of the utmost voltage control zones of the DG units.

$$
\begin{aligned}
& 0 \leq \text { Range } 1<R 1, R 1 \leq \text { Range } 2<R 2, R 2 \leq \text { Range } 3<R 3, \ldots . \\
& \forall R 1<R 2<R 3 \ldots
\end{aligned}
$$

Step-VII: Accordingly, the nodes within each range will determine the voltage control zone for a particular DG unit.

Step-VIII: The overlapping of voltage control zones can be avoided by appropriately classifying the overlapping nodes into each zone based on the respective electrical distances.

For example, a typical variation of DG voltage control zones of a distribution feeder system configuration similar to a system depicted in Fig. 1, where all the tie-lines are opened, which has two DG units (i.e., DG1 and DG2) is shown in Fig. 2. This is only a diagrammatic representation, which is based on the preliminary derivations of this case study. The end nodes of DG1 utmost voltage control zone are $\mathrm{N}_{\mathrm{a}}$ and $\mathrm{N}_{\mathrm{b}}$, where the end nodes of DG2 utmost voltage control zone are $\mathrm{N}_{\mathrm{c}}$ and $\mathrm{N}_{\mathrm{d}}$ along the feeder. According to the Step-VI, $R 1=e 1$, $R 2=e 2$ and $R 3=e 3$.

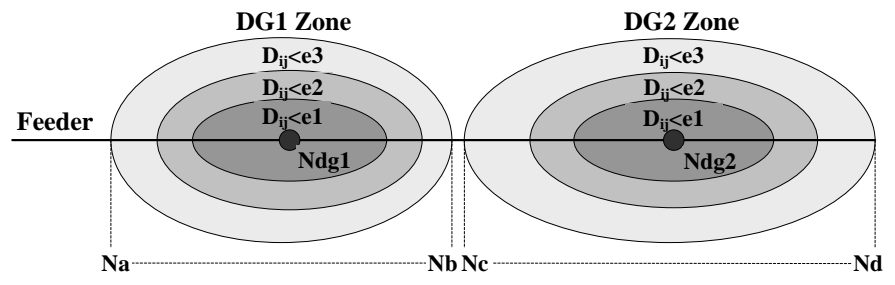

Fig. 2. Diagrammatic representation of DG voltage control zones for the sample distribution system configuration with DG1 and DG2.

If conventionally operated voltage regulating devices are located inside the DG zones, the operational aspects of those devices may be significantly detrimental, even leading to conflicting operations under certain system conditions. In each system configuration, the operation of SVRs (of Type-B type) can be demonstrated using their local control actions as given by (05) [10], [15]. The more common connection for SVRs is the Type-B. In Type-B connection, the primary of the SVR is connected to the series winding via taps; where the series winding is connected to the shunt winding which is connected to the SVR secondary [16].

$$
\begin{gathered}
\operatorname{Tap}_{s(t+1)}= \begin{cases}T_{a p}+1 & \text { if } V_{S L C}>V_{U B} \\
T_{s t} & \text { if } V_{L B} \leq V_{s L C} \leq V_{U B} \\
T a p_{s t}-1 & \text { if } V_{S L C}<V_{L B}\end{cases} \\
V_{L B}=V_{S E T / V R}-0.5 V_{D B} \text { and } V_{U B}=V_{S E T / V R}+0.5 V_{D B}
\end{gathered}
$$


The change of target point voltage of any $m^{\text {th }}$ SVR for system configuration, $s$ due to a small change in a DG unit reactive power response can be derived as given by (07).

$$
\Delta V_{s L C(m) / b y D G}=\left(M_{s V Q}\right)_{s L C(m), k} \times \Delta Q_{s D G}
$$

Similarly, in each system configuration, the operation of CBs can be demonstrated using their local control actions as given by (07) [10].

$$
S F C_{s(t+1)}= \begin{cases}O N & \text { if } S F C_{s t}=O F F \text { and } V_{S F C}<V_{O N} \\ S F C_{s t} & \text { if } V_{O N} \leq V_{S F C} \leq V_{O F F} \\ O F F & \text { if } S F C_{s t}=O N \text { and } V_{S F C}>V_{O F F}\end{cases}
$$

The change of target nodal voltage of any $g^{\text {th }}$ CB for system configuration, $s$ due to a small change in a DG unit reactive power response can be derived as given by (08). The target point voltage of the capacitor bank is normally its connection nodal voltage.

$$
S \Delta V_{S F C(g) / b y D G}=\left(M_{S V Q}\right)_{S C B(g), k} \times \Delta Q_{S D G}
$$

However, the following mathematical proof reveals that if the target node of a voltage regulating device is far from the node of a DG unit in terms of electrical distance, the impact of DG unit on the operation of the voltage regulating device is less and vice versa, under any system operation subjected to structural changes.

$$
\begin{array}{r}
\Delta V_{s i}=\mu_{s i k} \times \Delta V_{s k} \text { and } D_{\text {sik }}=-\log \left(\mu_{\text {sik }}\right) \\
\Delta V_{\text {silby } D G}=\left(\frac{1}{\log ^{-1}\left(D_{\text {sik }}\right)}\right) \times \Delta V_{\text {sk/by } D G} \quad \text { (09) } \\
\text { If } D_{\text {sik }} \rightarrow \propto, \log ^{-1}\left(D_{\text {sik }}\right) \rightarrow \propto, \text { then } \Delta V_{\text {silby } D G} \rightarrow 0 \\
\quad \text { From }(07),(08) \text { and }(09): \\
\Delta V_{s L C(m) / b y D G}, \Delta \mathrm{V}_{s F C(g) / b y D G} \rightarrow 0, \text { if } D_{s m k}, D_{s g k} \rightarrow \propto
\end{array}
$$

\section{Proposed Coordination Strategy}

The proposed coordination strategy is discussed in this section. The Section III $(A)$ outlines the sequence of operation of DG units and voltage regulating devices and Section III (B) is on proposed practical implementation strategy. The Section III (C) elaborates the algorithm of the proposed coordination strategy.

A. Sequence of Operation for DG Units and Voltage Regulating Devices

It is proposed to operate the DG units in VAr control mode thereby maximizing the voltage support. The VAr reference values of DG units are updated based on correcting the lowest nodal voltage (or highest voltage rise) by the most significant DG unit first, then by the next significant DG unit and so on. The most significant DG unit is identified by (a) using voltage control zones of the DG units and (b) electrical distance between DG units and the node which requires immediate voltage correction. For example, in case of a distribution system in Fig. 2, if the particular node which requires utmost voltage correction is within a particular DG zone (say DG2 zone), the VAr reference value of DG2 is updated first. If it is not adequate to maintain the voltage within the stipulated limits, the VAr reference value of next closer DG unit (in terms of electrical distance) i.e., DG1 is updated.

The sequence of operating voltage regulating devices (i.e., SVRs and CBs) is determined by prioritizing their operations based on the phenomenon demonstrated in (10) i.e., first operate the farthest voltage regulating device (with respect to DG units) in terms of electrical distance after updating the VAr reference values of the DG units, if the voltage is not within the stipulated limits. If the operation of farthest voltage regulating device is also not adequate to maintain the voltage within the stipulated limits, then operate the subsequent voltage regulating device, if available, which is located close to the DG units in terms of electrical distance and so on. Since, substation OLTC operates after a prolonged time delay (coarse control), it is not incorporated in the feeder voltage control strategy proposed in this paper. However, if needed, the operation of substation OLTC can also be incorporated in the proposed algorithm by incorporating the relevant upstream OLTCs and the voltage regulating devices in the medium voltage distribution network with DG.

\section{B. Online Implementation Strategy}

It is proposed to implement the proposed coordination scheme with the aid of a substation centered advanced distribution management system (DMS) for on-line voltage control [17], [18], [19]. The proposed control software and the hardware modules can be embedded in the DMS. The local control parameters of voltage regulating devices and DG units are tuned by a tuning algorithm embedded in the DMS which uses pseudo measurements of load and DG generation, and information on system structural changes as inputs. Since, network reconfiguration can be done online and availability of DG units can be monitored online with the aid of advanced DMS and associated infrastructure, the real-time status of structural changes is readily available. Also, the proposed voltage control strategy enhances the system security due to its capability to update control settings and enforce quick changeover to the local control of respective voltage regulating devices. The detailed topology of the proposed coordination module is shown in Fig. 3.

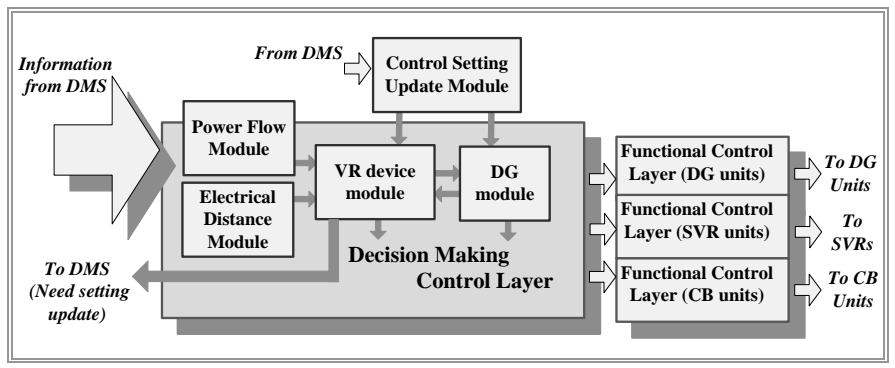

Fig. 3. Topology of the designed coordination module. 
The complete coordination module contains embedded power flow module, electric distance module, control setting update module, voltage regulating (VR) device module, DG module and the decision making control layer with separate functional control layers for DG units, SVRs and CBs for enacting those devices according to the information provided by the decision making control layer. The electric distance module is for determining the required electrical distance values from DG units to the corresponding voltage regulating devices subject to structural changes and availability of the DG units, and accordingly deriving the voltage control zones of DG units. The DG module is for determining the sequence of updating VAR reference values for DG units and VR device module is for determining the sequence of operation for VR devices (i.e., SVRs and CBs). The control setting updatemodule is for updating the control parameters of VR devices and DG units including VAr reference values of the DG units determined by tuning algorithm embedded in the DMS. Distribution system planning and operation by incorporating reactive power capabilities of different renewable and nonrenewable DG units is discussed in [20].

The control panels (CPs) of DG units and VR devices are proposed to be equipped with supervisory control and data acquisition (SCADA) facilities. This will have the functional capability of transmitting the real-time information about the status of VAr reference value update for DG units, SVR tap operations and CB switching operations to the coordination module via DMS. The edge-detection based methodology proposed by the authors in [1] is appropriately adopted for inferring real-time information about the status of VR devices and DG units. The VR devices and voltage support DG units are operated only after a confirmation from the coordination module i.e., by each functional control layer following the decision information taken by the decision making control layer. The control-algorithm embedded in the coordination module is summarized in the following sub section.

\section{Algorithm of the Proposed Coordination Strategy}

Step - 1: System structural changes, state estimation and power flow information are executed with the aid of relevant embedded DMS modules.

Step-2: The VAr reference values for DG units are determined by the tuning algorithm embedded in the DMS and assigned to coordination module via control setting updatemodule.

Step - 3: The sequence of operating DG units is determined by the DG module. The functions of power flow and electric distance modules and updated VAr reference values for DG units are called by the DG module for this task. If there are no structural changes in the system, the electric distance values of the previous state are used instead of calling and running the electric distance module again. The respective steps are outlined in the Section III $(A)$.

Step - 4: The sequence of operation for VR devices (i.e., SVRs and CBs) is determined by VR device module. The respective procedure is outlined using a flowchart in Fig. 4.
The functions of power flow and electric distance modules and updated VAr reference values for DG units are called by the VR device module whenever needed.

Step - 5: Based on the information given by the DG module and the VR device module (i.e., sequence of operation of DG units and VR devices) and the real-time information on the status of DG unit and VR device operations, the decision making control layer is enacted. Accordingly, the functional control layers for DG units, SVRs and CBs are enacted after the information from decision making control layer in order to operate the required device, only if the status of operation of the VR devices and the DG units do not lead to simultaneous responses. The functional control layers will pass this information to the respective CPs of the DG units and VR devices online and update for every new operation.

Step -6: For the subsequent instances of time (i.e., $t=t$ +1 ), repeat the procedure from Step -1 .

It is assumed that the load forecasts are known with sufficient accuracy via relevant modules of the DMS. Accordingly, the sensitivity of the proposed algorithm for constant power and constant impedance loads could be notable only if the sampling time between two consecutive control states is significantly large. In case of constant current loads, the electric distance values can further be updated using the algorithm embedded in voltage regulating device (VR) module for higher accuracy.

\section{Testing of the Proposed CoORdination StRategy}

The proposed coordination strategy is tested using the interconnected MV distribution system model derived from the distribution network of the state of New South Wales, Australia. This distribution system has long feeders, which are subjected to significant voltage drops. Balanced distribution feeder system operation (i.e., feeder configuration 01 with two DG units (DG1 and DG2) and feeder configuration 02 with one DG unit (DG2) are used as the test feeder systems, as shown in Fig. 5, in this paper for demonstrating the proposed coordination strategy. The feeder system associated with configuration- 01 is around $41.6 \mathrm{~km}$ long and has 95 nodes. It is fed by a $132 / 11 \mathrm{kV}, 30$ MVA transformer equipped with an OLTC. Also, two SVRs (Type-B type), SVR1 and SVR2, are installed at $25^{\text {th }}$ node and $51^{\text {th }}$ node of the test feeder system, respectively. Moreover, a switched shunt capacitor bank, CB1 of 0.50 MVAr capacity is connected at node-92. Synchronous machine based two bio-diesel generators (DG1 with 0.850 MVA capacity and DG2 with 1.000 MVA capacity) are connected to the distribution feeder system (one is at $38^{\text {th }}$ node and other is at $78^{\text {th }}$ node). There are 69 nodes in the feeder configuration-02, where total line length is around $30.4 \mathrm{~km}$. There is one SVR (SVR3) which has been connected at node no. 12 (of feeder configuration 02) in addition to SVR2, CB1 and DG2. According to the Australian standard for Standard Voltages (AS 60038-2012), the representative simulated voltage limits of the test system are $1.10 \mathrm{pu}$ and $0.90 \mathrm{pu}$. 


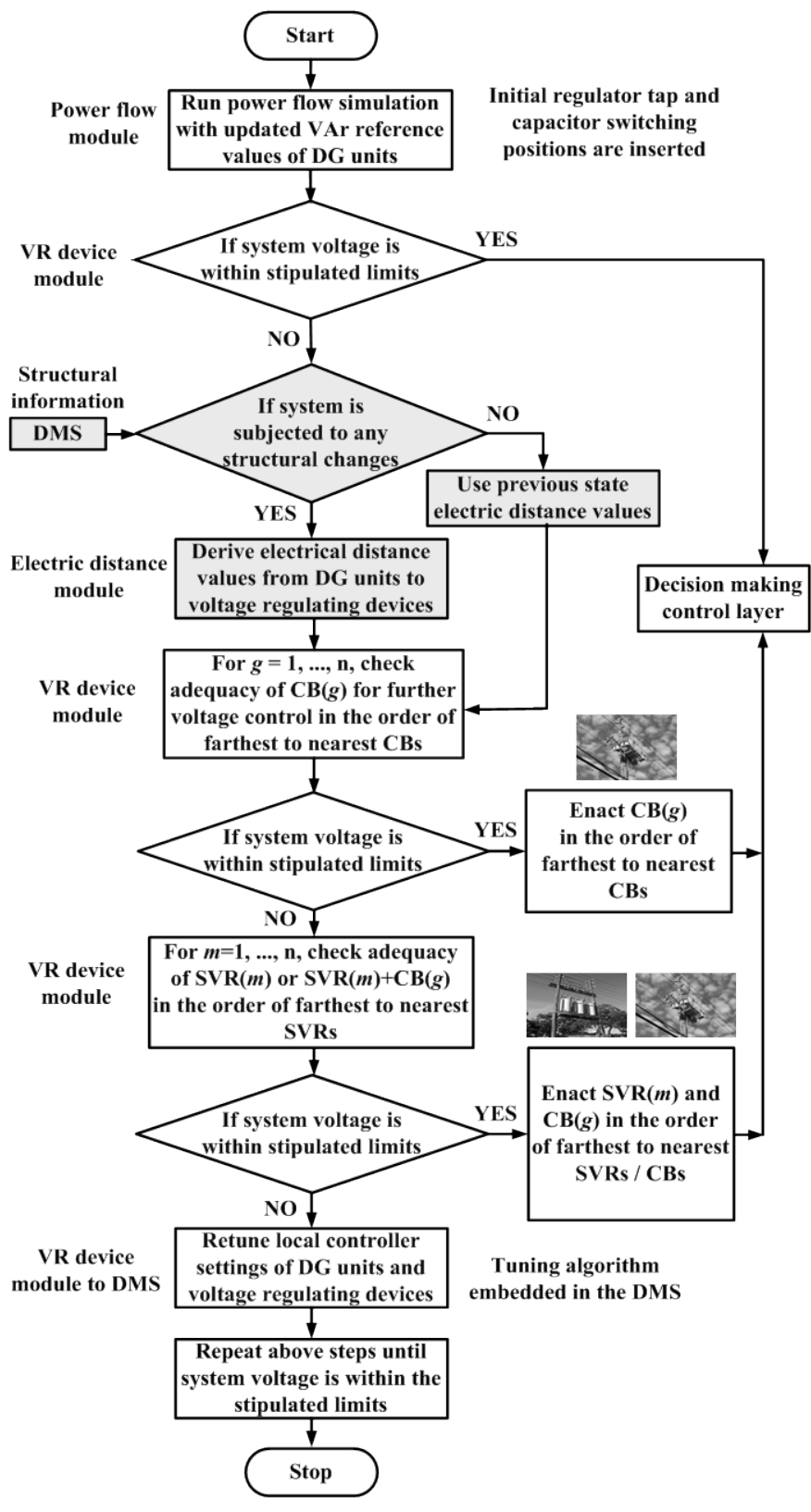

Fig. 4. Flowchart of the VR device module.

\section{A. Test Simulation for Feeder Configuration 01 with Two DG Units (DG1 and DG2)}

In this section, a MATLAB simulation is used to (a) demonstrate the phenomenon behind simultaneous and nonsimultaneous responses of DG units and VR devices and (b) implement the control action of the proposed coordination strategy to minimize their impacts. For this simulation, a system state (5 minute interval) with 3.0153 MVA representative total load demand with constant power characteristics and DG responses at different occasions of the test system are simulated. The simulated data of SVR1, SVR2 and CB1 are shown in Tables I and II.

The time delay (including the total mechanical time delay, is used only for the first tap operation of SVRs and the subsequent taps are operated with the mechanical time delay.
TABLE I

Simulated Data of Step Voltage REgulators

\begin{tabular}{c|c|c|c|c}
\hline SVR Unit & $\begin{array}{c}\text { Voltage Set } \\
\text { Value (pu) }\end{array}$ & $\begin{array}{c}\text { Dead Band } \\
(\%)\end{array}$ & $\begin{array}{c}\text { Time } \\
\text { Delay }(\mathrm{s})\end{array}$ & $\begin{array}{c}\text { Load } \\
\text { Center }\end{array}$ \\
\hline SVR1/SVR3 & 1.0 & \pm 1.0 & 30 & $\mathrm{R}_{\mathrm{L}}=\mathrm{X}_{\mathrm{L}}=0$ \\
\hline SVR2 & 1.0 & \pm 1.0 & 40 & $\mathrm{R}_{\mathrm{L}}=\mathrm{X}_{\mathrm{L}}=0$ \\
\hline
\end{tabular}

The simulated time delay for the SVR1/SVR3 is $6.0 \mathrm{~s}$ and SVR2 is $4.0 \mathrm{~s}$. The initial tap position of SVR1 is 3 and tap position of SVR2 is 1 in the direction of increasing the secondary voltage. The simulated time delay for the CB1 is 0.1 s. The initial switching position of CB1 is its nominal position. The initial voltage reference value is set at $1.05 \mathrm{pu}$ for OLTC.

TABLE II

SiMULATED DATA OF FEEDER CAPACITOR BANK

\begin{tabular}{c|c|c|c|c}
\hline CB Unit & $\begin{array}{c}\text { Capacity } \\
\text { (MVAr) }\end{array}$ & $\begin{array}{c}\text { Switch ON } \\
\text { Voltage (pu) }\end{array}$ & $\begin{array}{c}\text { Switch OFF } \\
\text { Voltage (pu) }\end{array}$ & $\begin{array}{c}\text { Time Delay } \\
\text { (s) }\end{array}$ \\
\hline CB1 & $\begin{array}{c}0.50 \\
\text { (5 Steps of } \\
\text { 0.10 MVAr) }\end{array}$ & 0.900 & 1.010 & 0.10 \\
\hline
\end{tabular}

The simulated active and reactive power responses (case01) of available DG units (i.e., DG1 and DG2) for (a) noncoordinated operation are $\mathrm{P}_{\mathrm{DG} 1}=0.60 \mathrm{MW}$ for $\mathrm{t}<60 \mathrm{~s}, \mathrm{P}_{\mathrm{DG} 1}=$ $0.74 \mathrm{MW}$ for $\mathrm{t} \geq 60 \mathrm{~s}, \mathrm{P}_{\mathrm{DG} 2}=0.70 \mathrm{MW}$ for $\mathrm{t}<48 \mathrm{~s}, \mathrm{P}_{\mathrm{DG} 2}=$ $0.62 \mathrm{MW}$ for $\mathrm{t} \geq 48 \mathrm{~s}$ and no reactive power as the DG units are operated at unity power factor as per the IEEE standard 1547: Standard for Interconnecting Distributed Resources with Electric Power Systems, and (b) for proposed operation are $\mathrm{Q}_{\mathrm{DG} 1}=0.37 \mathrm{MVAr}, \mathrm{Q}_{\mathrm{DG} 2}=0.43 \mathrm{MVAr}$ in addition to the same active power responses as indicated in part (a) earlier in this paragraph.

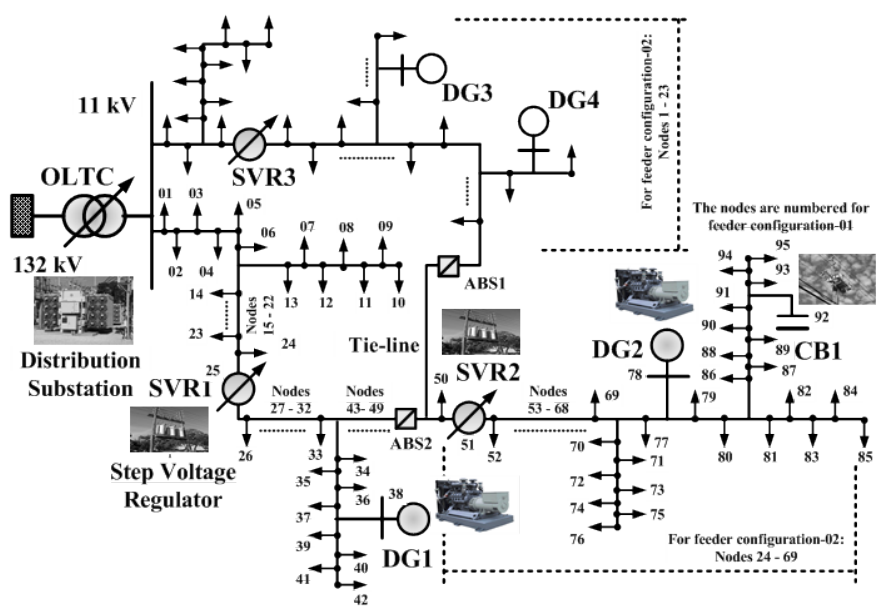

* Feeder Configuration - 01: System with ABS-1 opened and ABS-2 closed (OLTC, 2 SVRs, DG1, DG2 and CB are in operation)

* Feeder Configuration - 02: System with ABS-1 closed and ABS-2 opened (OLTC, 2 SVRs, DG2 and CB are in operation and DG3, DG4 are disconnected)

Fig. 5. Topology of the test distribution feeder system.

The simulated results for the non-coordinated operation [9] of VR devices and DG units are shown in Fig. 6. The Fig. 6 (a) is for case-01 and Fig. 6 (b) is for associated case where there is no change in the DG response (case-02). The normalized values of electrical distances to each VR device (a) from the DG1 unit are 0.0761 for SVR1, 0.0812 for SVR2, 0.1790 for CB1 and (b) from the DG2 unit are 0.1938 for SVR1, 0.0568 
for SVR2, 0.0292 for CB1. According to the control logic as given by (02) and subsequent time delay, the CB1 is operated first, up to its maximum switching position, supplying 0.50 MVAr. The total operational time is 0.4 s (i.e. time, $t=0$ to $0.4 \mathrm{~s})$. Next, according to the control logic as given by (01) and after the requisite time delays, SVR1 is operated at $\mathrm{t}=30$ $\mathrm{s}$ and SVR2 is operated at $\mathrm{t}=40 \mathrm{~s}$. In this case (case-01, Fig. 6 (a)), there are 8 tap operations for SVR1 and 13 tap operations for SVR2. Also, there are simultaneous tap operations at $t=48$ s, $60 \mathrm{~s}$ and $72 \mathrm{~s}$. The total number of operations for SVR and CB are 25. Moreover, the interactions caused by simultaneous responses of SVRs and DG units at $\mathrm{t}=60 \mathrm{~s}$ (i.e., 0.60 to 0.74 MW of DG1 in addition to 0.70 to $0.62 \mathrm{MW}$ of DG2 at $\mathrm{t}=48$ s) lead to violation of dead band limit of SVR1, because SVR1 is closer to the DG1 in terms of the electric distance where there is a fast response by DG1 at $t=60 \mathrm{~s}$. Hence tap operations (upward) are required for SVR1 to correct the voltage with respect to its voltage set value. In case of a light load scenario, this could be more significant. The impact of simultaneous responses of multiple VR devices and DG units on system voltage profile is thoroughly analyzed and discussed in [1]. However, since DG1 is closer to the SVR1 and DG2 is closer to the SVR2 in terms of electric distance, the fast responses by DG1 and DG2 lead to a lesser number of tap operations in SVR1 and significantly a higher number of tap operations in SVR2, respectively compared to the scenario where there is no change in DG responses at $\mathrm{t}=48 \mathrm{~s}$ and $60 \mathrm{~s}$ (case-02) i.e. 9 tap operations by SVR1 (from 3 to 12) and 6 tap operations by SVR2 (from 1 to 7). This is an example for assessing the impact of non-simultaneous responses of DG units and VR devices. In the worst case scenario, if simultaneous and/or non-simultaneous responses of VR devices and DG units lead to such conflicting operations in each system configuration and system state of a day as well as throughout a year of 365 days, their impact on wear and tear of VR devices and the system voltage profile can be significant. For example, if there will be 6 tap operations more in each state as shown in the simulation (i.e., in Fig. 6 (a) compared to Fig. 6 (b)), there could be 630,720 additional tap operations as well as resultant voltage transients and variations per year. The variations in the remote end voltage attributed to each operation of VR devices and DG responses for the case-01 (Fig. 6 (a)) are shown in Fig. 7.

The simulated results for the coordinated operation of VR devices and DG units using the proposed algorithm, outlined in the section III- $C$, are also shown, assuming zero propagation delay in communication channels, zero processing time of control software and hardware as well as zero error in load and DG forecasting. Fig. 8 (a) and Fig. 8 (b) show the operation of VR devices for case-01 and case-02 respectively, while Fig. 9 shows the variations in remote end voltage attributed to each operation of VR devices and DG responses for case-01. The sequence of operating DG units and VR devices are in the order of DG2 followed by DG1, SVR1 and finally CB1. The VAr reference value of DG2 is updated first after a time delay of $1 \mathrm{~s}$ (i.e., at $\mathrm{t}=1 \mathrm{~s}$ ) and next the VAr reference value of DG1 is updated after a subsequent time delay of $1 \mathrm{~s}$ (i.e., at $\mathrm{t}=2 \mathrm{~s}$ ).
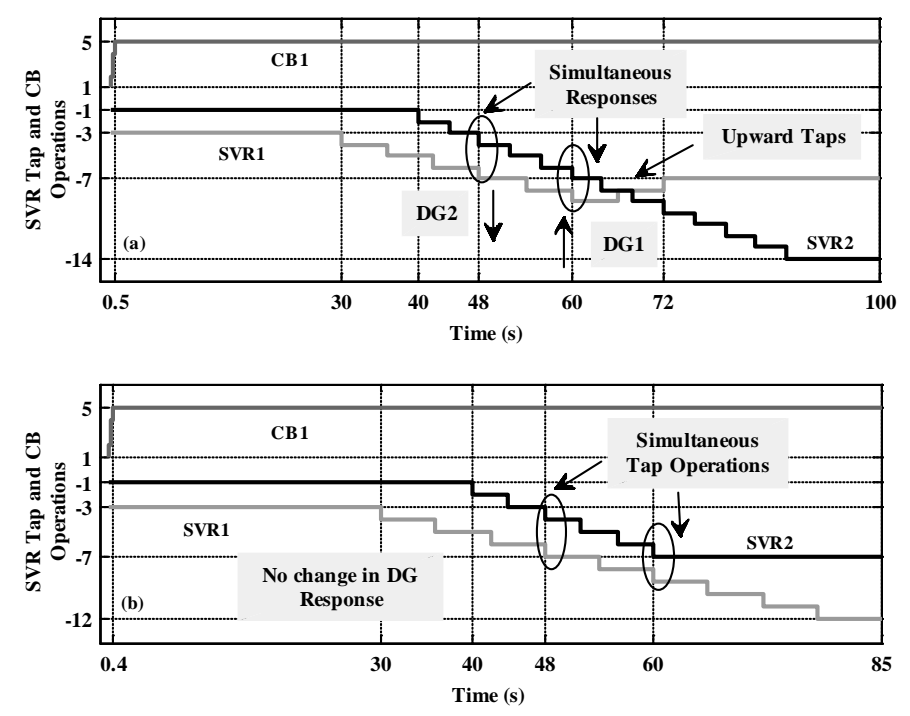

Fig. 6. Non-coordinated operation of voltage regulating devices in presence of DG for the simulated (a) case-01 and (b) case-02.

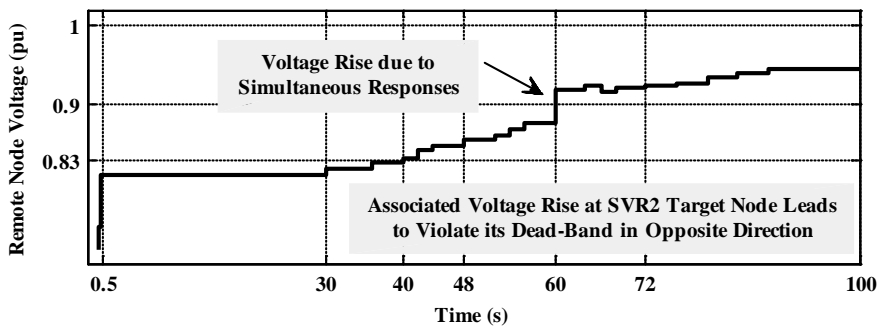

Fig. 7. Remote end voltage for the simulated case-01.

In this case, the total number of SVR tap operations is less compared to the scenario where there is no change in DG responses at $\mathrm{t}=48 \mathrm{~s}$ and $60 \mathrm{~s}$ (case-02) i.e., 9 tap operations. It is mainly because of the DG1 active power response at $\mathrm{t}=60$ $\mathrm{s}$, which significantly increases the voltage. However, still there is one tap operation (upward) in SVR1, since DG1 is closer to the SVR1 in terms of electric distance. Compared to the non-coordinated operation, only one upward tap operation of SVR1 is enacted to correct the voltage rise, and CB1 is operated after the SVR1 according to the proposed coordinated control algorithm. In case of the proposed coordinated operation, there are no simultaneous responses of DG units and VR devices. Also, the impact of interactions which can be caused by non-simultaneous responses of DG units and VR devices on the voltage profile is minimized, since the voltage regulating devices farthest to DG units (i.e., SVR1 and CB1 compared to SVR2) are operated on priority according to the electric distance.

The total number of SVR tap and CB switching operations (8 operations) are significantly less compared to the case of non-coordinated operation (25 operations) for the simulated state. If such an improvement is assumed in each system state throughout a year of 365 days, the annual reduction in VR device operations would be 1,787,040. 

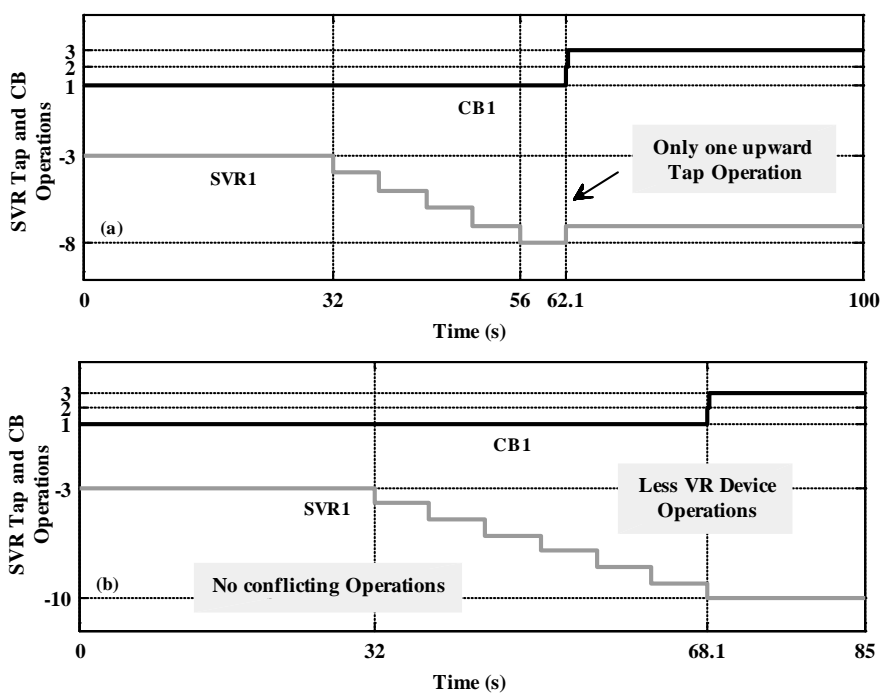

Fig. 8. Operation of VR devices for the simulated (a) case-01 and (b) case02 , under proposed coordination strategy.

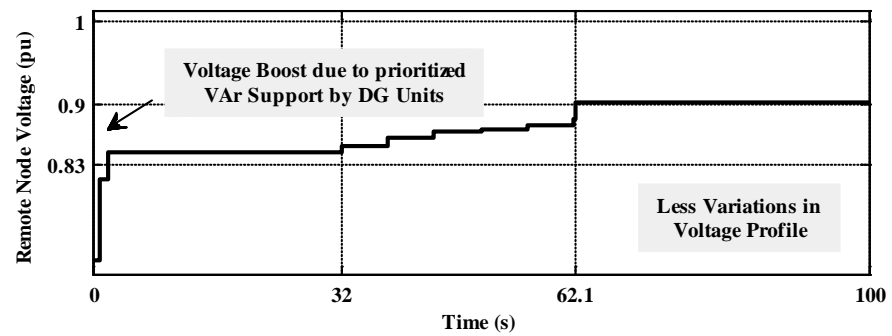

Fig. 9. Remote end voltage for the simulated case-01(a).

Reduction in tap and switching operations is indeed a key advantage of implementing the proposed voltage control strategy, which will obviously be beneficial to the distribution network operators as it directly correlates to the decrement in the regulator maintenance cost, increase in its life time expectancy, and the decrease in intermittent voltage variations attributed to the SVR tap and CB switching operations. It is noted that the simulated voltage profile in remote node of the test distribution feeder system is below the minimum voltage limit. These simulations are representative only and used to show the merits of the proposed method in regulating the voltage of the feeder end nodes with the aid of voltage regulating devices and minimizing the impact of their interactions on voltage profile where the system is subjected to structural changes. These simulations are useful in comparing the non-coordinated and proposed coordinated operations under worst case scenarios.

\section{B. Test Simulation for Feeder Configuration 02 with One DG Unit (DG2)}

In this section, the control action of proposed coordination strategy is further elaborated for above system configuration. The normalized values of electrical distances to each VR device from the DG2 unit are 0.3155 for SVR3, 0.1480 for SVR2 and 0.0360 for CB1. The simulated total load demand, $\mathrm{P}_{\mathrm{DG} 2}$ and $\mathrm{Q}_{\mathrm{DG} 2}$ are $3.4310 \mathrm{MW}, 0.65 \mathrm{MVAr}$ at $\mathrm{t}=0 \mathrm{~s}$ and 0.80 MW and 0.43 MVAr at $t=64 \mathrm{~s}$, respectively. The sequence of operating DG2 unit and VR devices is in the order of DG2 followed by SVR3 and finally SVR2 under proposed control strategy. Their non-coordinated and proposed coordinated operations are shown in Fig. 10 (a) and Fig. 11 (a) respectively, where the variations in the remote end voltage attributed to each operation of VR devices and DG responses are shown in Fig. 10 (b) and Fig. 11 (b) respectively. Under non coordinated voltage control, there are conflicting operations in SVR2 and CB1, since DG2 is electric distance wise closer to those devices.
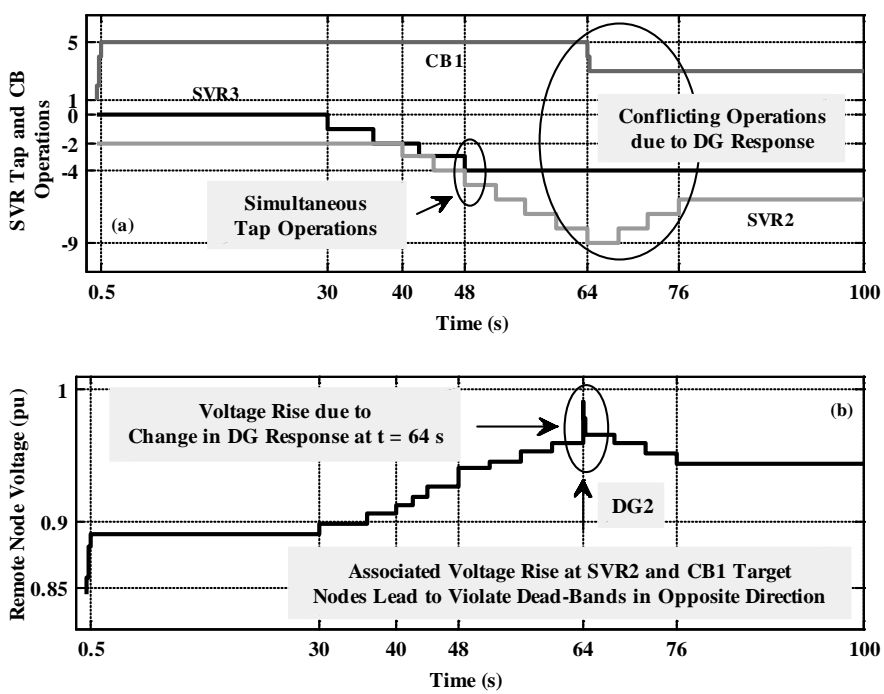

Fig. 10. (a) Operations for VR devices and (b) remote node voltage, under conventional non-coordinated voltage control.
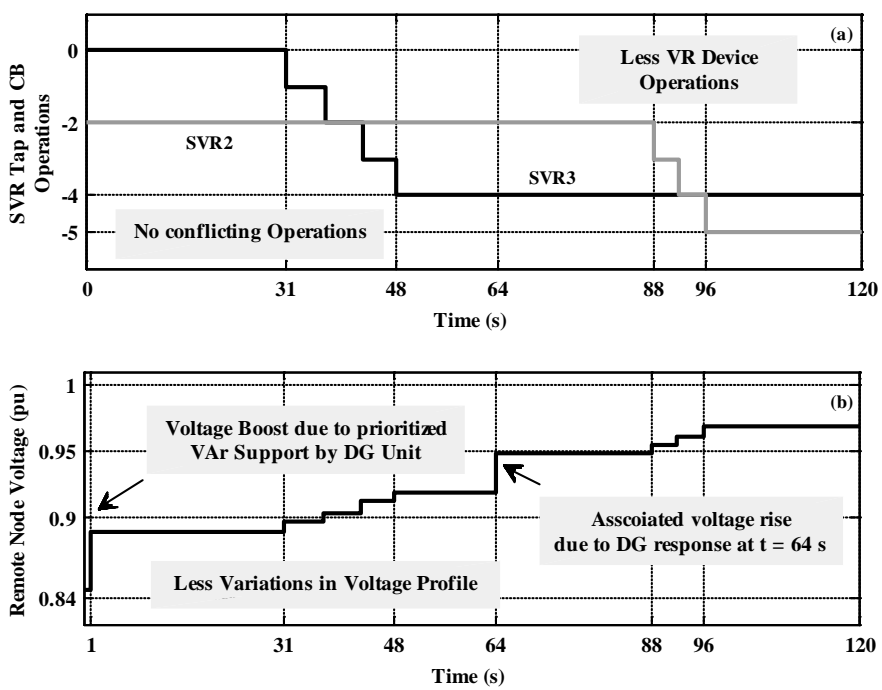

Fig. 11. (a) Operations for VR devices and (b) remote node voltage, under proposed coordinated voltage control.

In this simulated state, there is no requirement of operating CB1 under proposed coordinated voltage control. Also, there is no violation in the system voltage due to change in the DG2 response at $\mathrm{t}=64 \mathrm{~s}$ and there are less SVR2 tap operations. Moreover, it is noted if the SVR2 (SVR located downstream of the feeder) has to be operated first based on the electric distance values with reference to DG units; it will be enacted first unless there is any violation in system voltage after the 
operation of SVR1 and/or CB1. Otherwise, local controller settings of DG units and VR devices have to be re-tuned as mentioned in the flowchart of VR device module which is shown in Fig. 4, while maintaining the stipulated limit of voltage recovery time.

\section{Test Simulation under Real-time Environment for Feeder Configuration 01 with Two DG Units (DG1 and DG2)}

In this section, the real-time control action of proposed strategy is tested using separate online simulations for the different system states which are on 5-minute (300 s) interval basis. Fig. 12 shows the topology of the experimental setup. The real-world test distribution system topology is modeled using MATLAB SimuLink, where the system loads are modeled depicting variations in a daily load pattern. The local controllers of OLTC, SVRs, CB and DG units are modelled preserving their real-time operations. The proposed coordinated control model is implemented in MATLAB using separate MATLAB-functions for each module and the decision making control layer (as depicted in Fig. 3), which exhibit higher computational efficiency. The functional control layers (as depicted in Fig. 3), which give the appropriate signals to operate the required DG units and VR device local controllers are modeled with time delay embedded functions. The MATLAB script, ms.m is used for loading system parameters, setting up study conditions and plotting results. The proposed algorithm based information required for the functional control layers (i.e., confirmation to operate the device) is set by updating the time delay variable of each time delay embedded function (i.e. Embedded Function_1 to Embedded Function_4 as shown in Fig. 12); after running the MATLAB functions and based on their outputs. The on-line information to and from DG units, VR devices and distribution system are assigned by 'Goto' and 'From' signal blocks without propagation delay assuming zero communication delay.

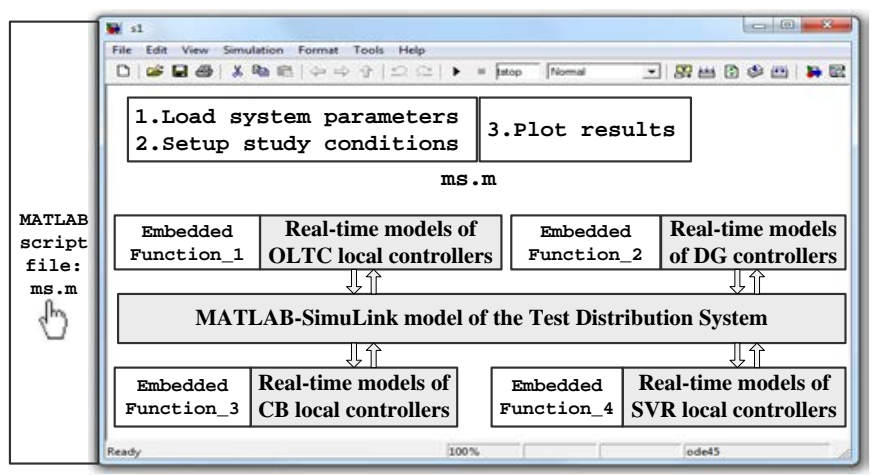

Fig. 12. Topology of the experimental setup used for test simulation under real-time environment.

Table III highlights the results obtained using one of the simulated demand patterns of the test distribution system for a system state at $300 \mathrm{~s}$. The daily peak load demand is also reflected in this scenario. The DG units are initially operated at unity power factor mode. The initial tap positions of SVR1 and SVR2 are 5 and 8, respectively in the direction of increasing the voltage. The initial switching position of CB1 is
2. Other simulated data of SVRs and CB1 remain the same as used in the respective steady-state network simulation. The proposed coordinated controller is executed for total load demand of 3.5090 MVA, and active power generation of 761.6 $\mathrm{kW}$ for DG1 and $641.3 \mathrm{~kW}$ for DG2. The new VAr reference values of DG1 and DG2 are $300 \mathrm{kVAr}$ and $450 \mathrm{kVAr}$, respectively and these values are updated with $1 \mathrm{~s}$ time delay. The operational sequence of DG units and VR devices under proposed coordinated control follows the sequential order of DG2, DG1, SVR1 and CB1. It is noted that for the simulated daily operation of this test feeder system (configuration - 01), the operations of only SVR1 and CB1 are adequate for voltage control with respect to VAR reference values of the DG units derived in the proposed coordination strategy.

TABLE III

SimUlated DEMAND PATTERN FOR TEST DISTRIBUTION SYSTEM

\begin{tabular}{c|c|c|c|c|c}
\hline Time (s) & 0 & 60 & 120 & 180 & 240 \\
\hline $\begin{array}{c}\text { (\%) Demand } \\
\text { to peak value }\end{array}$ & $89.8 \%$ & $94.6 \%$ & $97.8 \%$ & $100 \%$ & $96.4 \%$ \\
\hline
\end{tabular}

The SVR tap and CB switching operations under noncoordinated [9] and proposed coordinated voltage control are shown in Fig. 13 (a) and Fig. 13 (b), respectively. The respective nodal voltage profiles for feeder operation with non-coordinated voltage control and proposed voltage control are shown in Fig. 14 and Fig. 15.

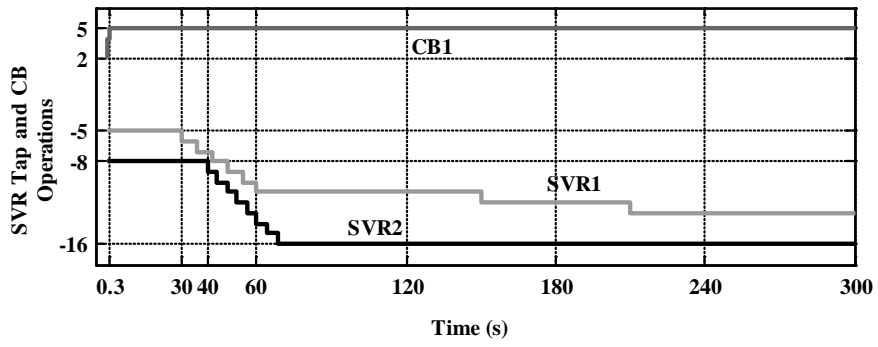

(a)

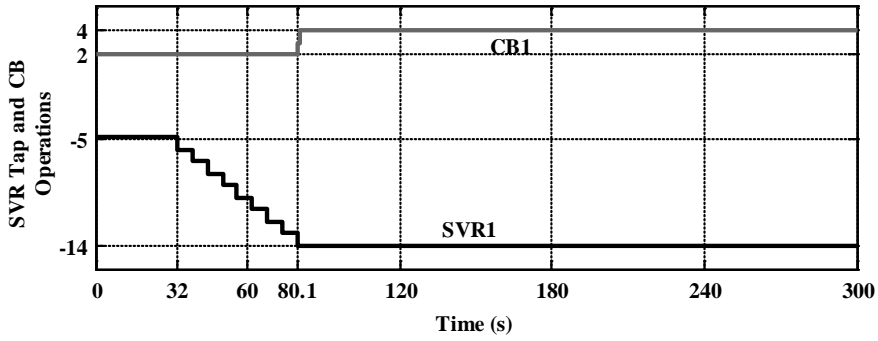

(b)

Fig. 13. Operation of VR devices for the simulated state under (a) noncoordinated voltage control (b) proposed coordinated voltage control.

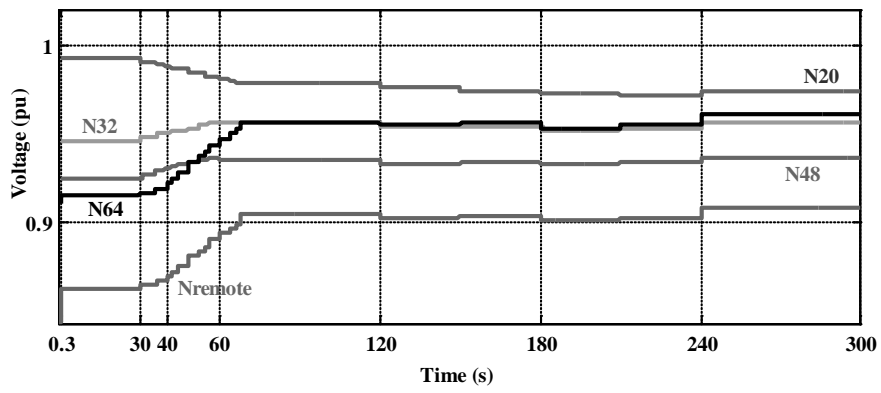

Fig. 14. Voltage at selected nodes for test distribution feeder operation under non-coordinated voltage control. 


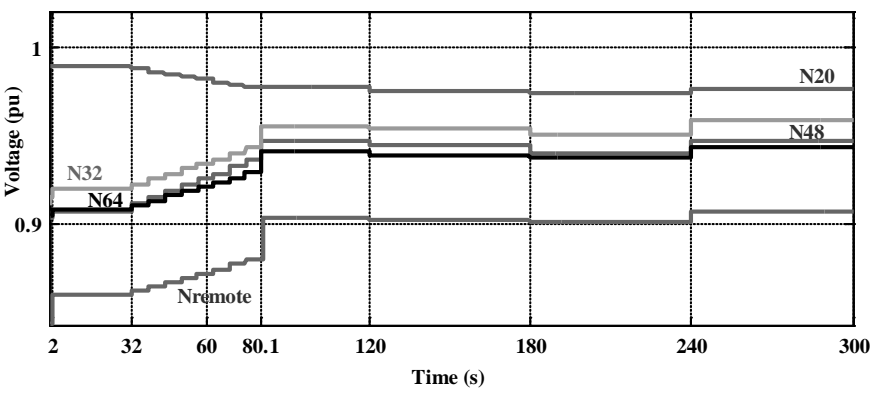

Fig. 15. Voltage at selected nodes for proposed coordinated voltage control.

There are simultaneous tap operations at $\mathrm{t}=48 \mathrm{~s}$ and $\mathrm{t}=60$ $\mathrm{s}$, and SVR2 reaches maximum tap position in the direction of increasing the voltage under non-coordinated operation. Also, there are additional tap operations of SVR1 at $\mathrm{t}=150 \mathrm{~s}$ and $\mathrm{t}=$ $210 \mathrm{~s}$ for correcting the voltage. The effect of proposed coordinated voltage control strategy to regulate the voltage of test distribution feeder system can be observed in the selected nodes, which are node-20 (between substation and SVR1), node-32 (between SVR1 and DG1), node-48 (between DG1 and SVR2), node-64 (between SVR2 and DG2), and nodeN95 (at the remote end).

\section{CONCLUSION}

This paper proposes a strategy for coordinating multiple VR devices and DG units in a MV distribution system with structural changes and DG availability, thereby improving their overall voltage control performance. It coordinates the operation of multiple VR devices and DG units, and operates the DG units on a priority basis for maximizing the voltage support by DG, in order to minimize the impact of interactions which can be caused by simultaneous and non-simultaneous responses of VR devices and DG systems. The proposed priority scheme can improve the system voltage profile by eliminating uncoordinated operation of VR devices and DG thereby reducing possible conflicting operations and resultant voltage variations in the system. The proposed coordination algorithm is based on the structural information derived by adopting electrical distance estimation, which is used to determine the sequence of operation for DG units and VR devices. Therefore, it effectively assesses the impact of structural changes in the network on the control interactions.

\section{REFERENCES}

[1] D. Ranamuka, A. P. Agalgaonkar, and K. M. Muttaqi, “On-line Voltage Control in Distribution Systems with Multiple Voltage Regulating Devices,” IEEE Trans. Sustainable Energy, vol. 5, no. 2, pp. 617-628, Apr. 2014.

[2] A. Kulmala, S. Repo, and P. Jarventausta, "Coordinated Voltage Control in Distribution Networks Including Several Distributed Energy Resources,” IEEE Trans. Smart Grid, vol. 5, no. 4, pp. 2010-2020, Jul. 2014.

[3] K. Alobeidli, and M. S. E. Moursi, "Novel Coordinated Secondary Voltage Control Strategy for Efficient Utilisation of Distributed Generations,” IET Renewable Power Generation, vol. 8, no. 5, pp. 569579, Jan. 2014.

[4] M. S. E. Moursi, H. H. Zeineldin, J. L. Kirtley, and K. Alobeidli, “A Dynamic Master/Slave Reactive Power-Management Scheme for Smart
Grids with Distributed Generation,” IEEE Trans. Power Delivery, vol. 29, no. 3, pp. 1157-1167, Jun. 2014.

[5] X. Liu, A. Aichhorn, L. Liu, and H. Li, "Coordinated Control of Distributed Energy Storage System With Tap Changer Transformers for Voltage Rise Mitigation Under High Photovoltaic Penetration,” IEEE Trans. Smart Grid, vol. 3, no. 2, pp. 897-906, Jun. 2012.

[6] M. E. Elkhatib, R. E. Shatshat, and M. M. A. Salama, "Novel Coordinated Voltage Control for Smart Distribution Networks with DG,” IEEE Trans. Smart Grid, vol. 2, no. 4, pp. 598-605, Dec. 2011.

[7] L. Yu, D. Czarkowski, and F. D León, "Optimal Distributed Voltage Regulation for Secondary Networks with DGs," IEEE Trans. Smart Grid, vol. 3, no. 2, pp. 959-967, Jun. 2012.

[8] S.C.E. Jupe P.C. Taylor, and A. Michiorri, "Coordinated Output Control of Multiple Distributed Generation Schemes," IET Renewable Power Generation, vol. 4, no. 3, pp. 283-297, Jan. 2010.

[9] F. Bignucolo, R. Caldon, and V. Prandoni, "Radial MV Networks Voltage Regulation with Distribution Management System Coordinated Controller," Electric Power Systems Research, vol. 78, no. 4, pp. 634645, Apr. 2008.

[10] F. A. Viawan, and D. Karlsson, "Combined Local and Remote Voltage and Reactive Power Control in the Presence of Induction Machine Distributed Generation,” IEEE Trans. Power Systems, vol. 22, no. 4, pp. 2003-2012, Nov. 2007.

[11] T. Senjyu Y. Miyazato, A. Yona, N. Urasaki, and T. Funabashi, "Optimal Distribution Voltage Control and Coordination With Distributed Generation,” IEEE Trans. Power Delivery, vol. 23, no. 2, pp. 1236-1242, Apr. 2008.

[12] Y. J. Kim, S. J. Ahn, P. I. Hwang, G. C. Pyo, and S. I. Moon, "Coordinated Control of a DG and Voltage Control Devices Using a Dynamic Programming Algorithm," IEEE Trans. Power Systems, vol. 28, no. 1, pp. 42-51, Feb. 2013.

[13] J. Zhong, E. Nobile, and K. Bhattacharya, "Localized Reactive Power Markets Using the Concept of Voltage Control Areas," IEEE Trans. Power Systems, vol. 19, no. 3, pp. 1555-1561, Aug. 2004.

[14] P. Lagonotte, J. C. Sabonnadiere, J. Y. Leost, and J. P. Paul, "Structural analysis of the electrical system: Application to secondary voltage control in France," IEEE Trans. Power Systems, vol. 4, no. 2, pp. 479486, May 1989.

[15] P. Kundur, Power System Stability and Control, vol. 1. New York: McGraw-Hill, 1994, p. 683-687.

[16] W. H. Kersting, Distribution System Modeling and Analysis, vol. 1. Boca Raton: CRS Press, 2002, p. 204.

[17] NR Electric Corporation. Distribution Management System. [Online]. Available: http://www.nrelect.com

[18] B. Uluski, "Distribution Management Systems," presented at the CRN Summit, Cleveland Ohio, 2011.

[19] ABB Pvt. Ltd. Network Manger SCADA/DMS Distribution Network Management. [Online]. Available: http://www.abb.com

[20] K. Zou, A. P. Agalgaonkar, K. M. Muttaqi, and S. Perera, "Distribution System Planning With Incorporating DG Reactive Capability and System Uncertainties,” IEEE Trans. Sustainable Energy, vol. 3, no. 1, pp. 112-123, Jan. 2012. 